\section{OP0257-PARE USING PATIENT HEALTH LITERACY PROFILES TO IDENTIFY SOLUTIONS TO CHALLENGES FACED IN RHEUMATOLOGY CARE}

M. Bakker ${ }^{1}$, P. Putrik ${ }^{1}$, J. Rademakers ${ }^{2}$, M. Van de Laar ${ }^{3}$, H. Vonkeman ${ }^{3}$, M. R. Kok ${ }^{4}$, H. Voorneveld ${ }^{4}$, S. Ramiro ${ }^{5}$, M. De Wit ${ }^{6}$, R. Osborne ${ }^{7}$, R. Batterham ${ }^{7}$, R. Buchbinder ${ }^{8}$, A. Boonen ${ }^{1} .{ }^{1} M U M C+$, Maastricht, Netherlands; ${ }^{2}$ Nivel, Utrecht, Netherlands; ${ }^{3}$ MST, Enschede, Netherlands; ${ }^{4}$ Maasstad Hospital, Rotterdam, Netherlands; ${ }^{5}$ LUMC, Leiden, Netherlands; ${ }^{6}$ AUMC, Amsterdam, Netherlands; ${ }^{7}$ Swinburne University, Melbourne, Australia; ${ }^{8}$ Monash University, Melbourne, Australia

Background: The prevalence of limited health literacy (i.e. cognitive and social resources of individuals to access, understand and apply health information to promote and maintain good health) in the Netherlands is estimated to be over $36 \%$ [1]. Access to and outcomes of rheumatological care may be compromised by limited patient health literacy, yet little is known about how to address this, thus action is required. As influencing individual patients' health literacy in the rheumatology context is often unrealistic, it is paramount for the health system to be tailored to the health literacy needs of its patients. The OPtimising HEalth LIteracy and Access (Ophelia) process offers a method to inform system change [2]. Objectives: Following the Ophelia approach:

a. Identify health literacy profiles reflecting strengths and weaknesses of outpatients with RA, SpA and gout.

b. Use the health literacy profiles to facilitate discussions on challenges for patients and professionals in rheumatological care and identify possible solutions the health system could offer to address these challenges.

Methods: Patients with RA, SpA and gout attending outpatient clinics in three centres in the Netherlands completed the Health Literacy Questionnaire (HLQ) and questions on socio-demographic and health-related characteristics. Hierarchical cluster analysis using Ward's method identified clusters based on the nine HLQ domains. Three researchers jointly examined 24 cluster solutions for meaningfulness by interpreting HLQ domain scores and patient characteristics. Meaningful clusters were translated into health literacy profiles using HLQ patterns and demographic data. A patient research partner confirmed the identified profiles. Patient vignettes were designed by combining cluster analyses results with qualitative patient interviews. The vignettes were used in two two-hour co-design workshops with rheumatologists and nurses to discuss their perspective on health literacy-related challenges for patients and professionals, and generate ideas on how to address these challenges.

Results: In total, 895 patients participated: $49 \%$ female, mean age 61 years $( \pm 13.0), 25 \%$ lived alone, $18 \%$ had a migrant background, $6.6 \%$ did not speak Dutch at home and $51 \%$ had low levels of education. Figure 1 shows a heat map of identified health literacy profiles, displaying the score distribution per profile across nine health literacy domains. Figure 2 shows an excerpt of a patient vignette, describing challenges for a patient with profile number 9 . The workshops were attended by 7 and 14 nurses and rheumatologists. Proposed solutions included health literacy communication training for professionals, developing and improving (visual) patient information materials, peer support for patients through patient associations or group consultations, a clear referral system for patients who need additional guidance by a nurse, social worker, lifestyle coach, pharmacist or family doctor, and more time with rheumatology nurses for target populations. Moreover, several system adaptations to the clinic, such as a central desk for all patient appointments, were proposed.

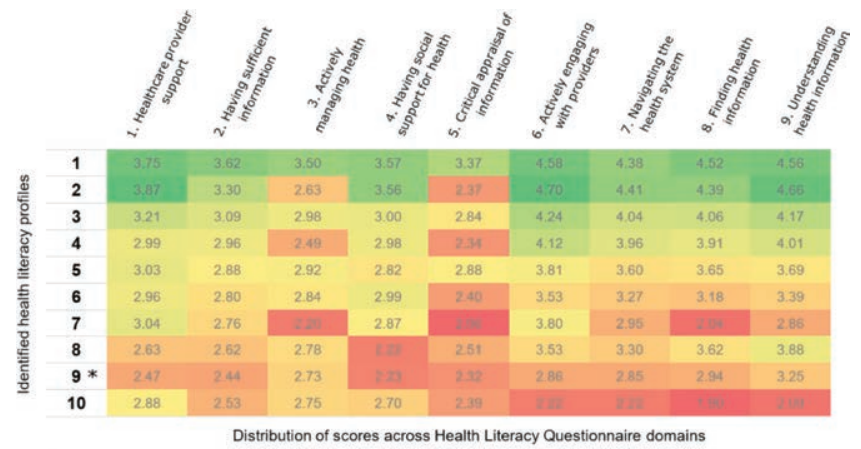

Figure 1. Heat map of identified health literacy profiles, with patterns of scores across nine health literacy domains. Range for domains $1-5$ is [1-4]; Range for domains 6-9 is [1-5]. Orange and red scores represent low to very low levels, yellow and green scores represent moderate to good levels. " indicates the profile displayed in figure 2.

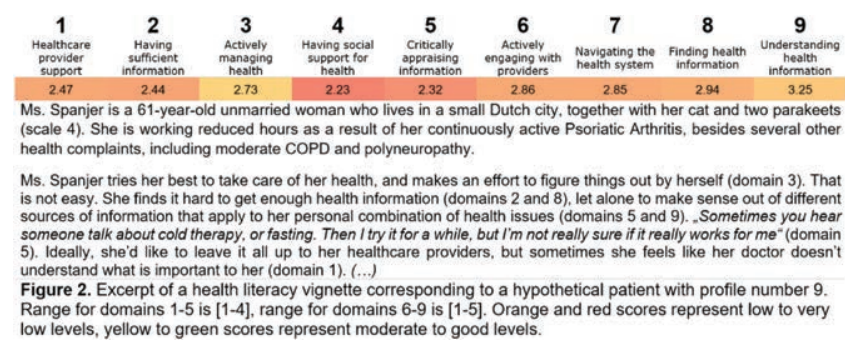

Conclusion: This study identified several distinct health literacy profiles of patients with rheumatic conditions. Engaging with health professionals in co-design workshops led to numerous bottom-up ideas to improve care. Next steps include co-design workshops with patients, followed by prioritising and testing proposed interventions.

\section{References:}

[1] Heijmans M. et al. Health Literacy in the Netherlands. Utrecht: Nivel 2018

[2] Batterham R. et al. BMC Public Health 2014, 14:694

Disclosure of Interests: Mark Bakker: None declared, Polina Putrik: None declared, Jany Rademakers Speakers bureau: In March 2017, Prof. Dr. Rademakers was invited to speak about health literacy at the "Heuvellanddagen" Conference, hosted by Janssen-Cilag., Mart van de Laar Consultant of: Sanof Genzyme, Speakers bureau: Sanofi Genzyme, Harald Vonkeman: None declared, Marc R Kok Grant/research support from: BMS and Novartis, Consultant of: Novartis and Galapagos, Hanneke Voorneveld: None declared, Sofia Ramiro Grant/research support from: MSD, Consultant of: Abbvie, Lilly, Novartis Sanofi Genzyme, Speakers bureau: Lilly, MSD, Novartis, Maarten de Wit Grant/ research support from: Dr. de Wit reports personal fees from Ely Lilly, 2019, personal fees from Celgene, 2019, personal fees from Pfizer, 2019, personal fees from Janssen-Cilag, 2017, outside the submitted work., Consultant of: Dr. de Wit reports personal fees from Ely Lilly, 2019, personal fees from Celgene, 2019 personal fees from Pfizer, 2019, personal fees from Janssen-Cilag, 2017, outside the submitted work., Speakers bureau: Dr. de Wit reports personal fees from Ely Lilly, 2019, personal fees from Celgene, 2019, personal fees from Pfizer, 2019 personal fees from Janssen-Cilag, 2017, outside the submitted work., Richard Osborne Consultant of: Prof. Osborne is a paid consultant for pharma in the field of influenza and related infectious diseases., Roy Batterham: None declared, Rachelle Buchbinder: None declared, Annelies Boonen Grant/research support from: AbbVie, Consultant of: Galapagos, Lilly (all paid to the department) DOI: 10.1136/annrheumdis-2020-eular.877

\section{OP0258-PARE ESTABLISHMENT AND DEPLOYMENT OF PATIENT EXPERT PROGRAM}

\section{A. Phoka Charalambous ${ }^{1} .{ }^{1}$ Cyprus League Against Rheumatism, Nicosia, Cyprus}

Background: Patient Expert on RMD's is a Patient Instructor who can train on the clinical examination technique of hand, hip, or knee for Rheumatoid Arthritis or Osteoarthritis. This Patient can explain the differences between RA and OS can give information about symptoms for RMDs, and can share personal experience. Having a Patient Expert Program in a patient organisation helps on Raising awareness with future Health Professionals, gives strength and makes a stronger voice. After a very successful development of the Patient Expert Program, with the help of Eular Pare Knowledge Transfer Program, the time for installation of the program in universities has come.

Objectives: The aim was Medical Schools and Health Professional Universities to accept the program, in order for it to be a part of their education or training program. Having the program as part of their education or training program would benefit the next generation of Physicians or Health Professionals in the following ways. Better understanding for RMD's and the patient perspective, increaseing awareness and early diagnosis, to build a rapport with the patient. The challenge was in having the program accepted by the schools and universities, as is the only program on this kind in the country.

Methods: We created a task force to achieve our goal. The task force developed a strategic plan using the Swot analysis, to be able to achieve our goal. From that analysis the following plan was made: First we needed to prepare a presentation for the program. Second, we needed to demostrate it. Third, we had to have all our evaluation forms ready. And lastly, we had to make contacts with Medical schools by writing and by calling them. The stronger weakness and threat that came up was the possible refusal of the Universities, to accept the program. Results: We started our communications with the Medical Schools when all of the steps were ready. The Medical School that had undergraduate students accepted to have a meeting with us. During our meeting, we made the oral presentation of our program and the Professors' response was positive and they proposed it to the Academic Board. The Academic Board asked for a demostration 
and so we did. In the end, the Academic Board accepted our proposal. The program runs on students in the fourth Academic Year, in small groups, and on the premises of the school. The other one with postgraduate Medical Students didn't accept the proposal of our program, with the justification that as a department of a foreign Medical school, they couldn't implement anything in the students program. So far, more than 10 trainings have been conducted. At the end of the first year that the program was implemented, the Medical School asked from the organisation for a Patient Instructor to take part at the Musculoskeletal Examination and be a model for the Examination. Seeing our success, and taking into consideration the students' evaluation of the program, especially the fact that $95 \%$ of them said, 'It was very important for them to have a Patient Expert Program during their studies' our organisation decided to expland our collaboration with more Healthcare Professions Universities. Our original goal had been to collaborate with one of the medical schools/universities, but now we have bigger plans. Now we want this program to be established in all the universities in the country with the Patient Expert RMD's program implemented in all health professional curriculums.

Conclusion: The importance of the Patient Expert in RMD's is unquestionable. Patient Expert Program is an excellent tool to raise awareness on RMD's. Also it is very helpful for the Medical or Health care Professions student as it can help them better understand the disease and the patients. The success of the program is also giving motivation to the organisation to expand and improve the program. It also shows the significance of the program for the next generation of Physicians and Healthcare Professionals. Our organisation is leading in patient centered care and is proof that making the patient the most important is the target of treatment for RMD's in our country.

Disclosure of Interests: None declared

DOI: 10.1136/annrheumdis-2020-eular.2111

\section{OP0259-PARE WORLD YOUNG RHEUMATIC DISEASES (WORD) DAY: THE FIRST INTERNATIONAL AWARENESS DAY FOR PAEDIATRIC RHEUMATIC DISEASES}

S. Stones ${ }^{1}$, E. Smith ${ }^{2}$, S. Ainsworth ${ }^{1}$, V. Buys ${ }^{1}$, W. Costello ${ }^{1}$, Y. Egert ${ }^{1}$ H. Foster ${ }^{3}$, L. Lamot ${ }^{4}$, B. J. Prakken ${ }^{5}$, C. Scott ${ }^{6}$. ${ }^{1}$ European Network for Children with Arthritis, Geneva, Switzerland; ${ }^{2}$ University of Liverpool, Department of Women's and Children's Health, Liverpool, United Kingdom; ${ }^{3}$ Newcastle University Medicine Malaysia, Johor Bahru, Malaysia; ${ }^{4}$ Sestre Milosrdnice University Hospital Center and University of Zagreb School of Medicine, Department of Pediatrics, Zagreb, Croatia; ${ }^{5}$ University Medical Center Utrecht, Department of Pediatric Immunology, Utrecht, Netherlands; ${ }^{6}$ University of Cape Town/Red Cross War Memorial Children's Hospital, Paediatric Rheumatology, Cape Town, South Africa

Background: There is a lack of awareness of paediatric rheumatic diseases (PRDs), among the public, and certain groups of healthcare professionals, such as general practitioners [1]. To help improve awareness and understanding of PRDs, World Young Rheumatic Diseases Day (WORD Day) was established in 2019.

Objectives: The aim of WORD Day, which took place on 18 March 2019, was to raise awareness of PRDs, while informing young people, families, healthcare professionals, teachers, and the public about the importance of timely referral plus early diagnosis and access to appropriate treatment and support.

Methods: A steering committee was established for the inaugural campaign. An external agency was appointed to provide digital support, with an official social media campaign launching in December 2018. Social media analytics were used to measure the impact of official social media platforms. A range of branded materials were also made available (Fig 1), including an official campaign video. In addition, a specific engagement activity (\#ButtonChallenge2019) was launched. This challenged participants to button up a piece of clothing while wearing gloves, to simulate the difficulties that young people with PRDs face undertaking daily activities. As part of the campaign launch, a list of suggested activities were provided, along with template press releases.

Results: Several face-to-face and virtual events took place globally on or around WORD Day 2019, with 34 countries reporting events (Fig 2). Examples of events included lectures, social gatherings and media appearances. An official WORD Day tweet chat was also hosted. Regarding social media impact, between December 2018 and March 2019, a total of 2,585 and 660 individuals followed the official Facebook and Twitter accounts, respectively. Facebook posts were seen 646,000 times since the start of the campaign, with over 60,000 of Facebook reach from posts published on 18 March 2019 exclusively. A total of 270,800 impressions were observed on Twitter. The official \#WORDDay2019 hashtag was seen by 533,955 unique accounts on 18 March 2019 alone, with 3,334,699 impressions. Posts with the hashtag were retweeted 1,112 times on WORD Day, with a total of 1,568 tweets recorded that day. With regards to the \#ButtonChallenge2019, the challenge video was viewed for 6,700 minutes and received participation from across the world. Compared to the industry benchmark, the average engagements per post for WORD Day-related content was significantly higher compared to other medical and non-for-profit social media pages.

Conclusion: WORD Day 2019 was the first international campaign focused solely on PRDs. Organic and paid social media content aided the dissemination of the WORD Day message, with Facebook proving to be the most popular social platform. Despite a wealth of different content published, authentic materials, namely video content, proved to be the most popular with users, particularly when it featured material designed by and with young people with PRDs. It was demonstrated that awareness events can often be resource-light and easily implemented across a range of diverse countries. It is anticipated that the global reach of WORD Day will increase over time as the campaign becomes more established.

References:

[1] Egert $Y$ et al. Children and young people get rheumatic disease too. The Lancet Child \& Adolescent Health. 2019;3(1):8-9.
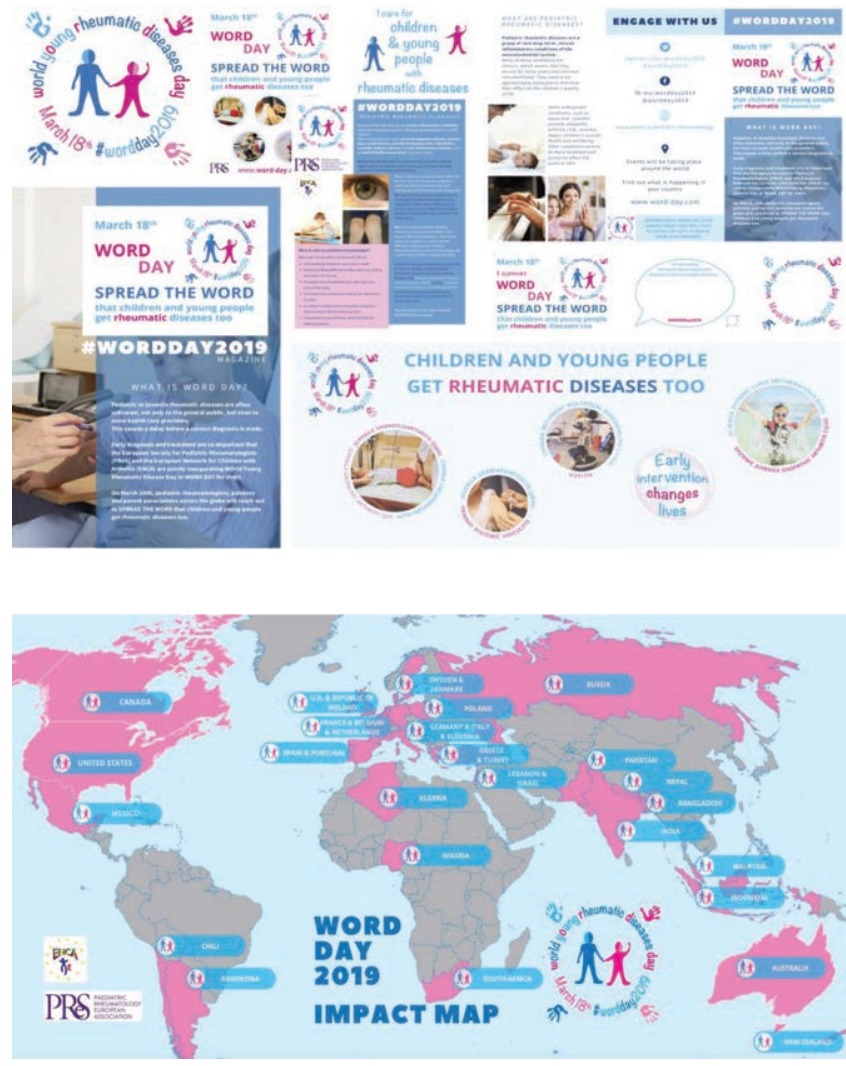

Acknowledgments: PReS for their financial support.

Disclosure of Interests: Simon Stones Consultant of: I have been a paid consultant for Envision Pharma Group and Parexel. This does not relate to this abstract. Speakers bureau: I have been a paid speaker for Actelion and Janssen. These do not relate to this abstract., Eve Smith: None declared, Sammy Ainsworth None declared, Veerle Buys: None declared, Wendy Costello: None declared Yona Egert: None declared, Helen Foster: None declared, Lovro Lamot: None declared, Berent J. Prakken: None declared, Christiaan Scott: None declared DOI: 10.1136/annrheumdis-2020-eular.1794

\section{OP0260-PARE EXPERIENCE EXCHANGE - FUNDRAISING AND PATIENT EXPERT PROGRAM}

\section{N. Nedić ${ }^{1}$ ' Serbia, Belgrade, Serbia}

Background: Since the establishment of ORS, we have been organising different activities for rheumatic diseases patients and their supporters within our five branches. After celebrating our $10^{\text {th }}$ anniversary in 2017 we were keen to expand and improve our activities, learning from an organisation with long tradition, in order to strengthen our position and to start reaching another level - growing from a small to a medium-sized organization in the next three years.

The main source of our income were the pharmaceutical companies (94\%). We wanted to reduce their contribution to our budget by $20 \%$ in the next three years. We had established good cooperation with two medical high schools and we would like to consider cooperation with a medical faculty. We were keen to gain insight into the Patient Expert Programme (PEP) project that Swedish Rheumatism Association had developed and hoped to then begin and steadily develop 